\title{
煅烧温度对 $\mathrm{Li}_{0.33} \mathrm{La}_{0.56} \mathrm{TiO}_{3}$ 固态离子电容器性能的影响
}

\author{
卢东亮 ${ }^{1,2}$, 代广周 ${ }^{1}$ ，姚英邦 ${ }^{1}$, 陶 涛 ${ }^{1}$, 梁 波 $^{1}$ ，鲁圣国 ${ }^{1}$
}

(1. 广东工业大学材料与能源学院, 广东省智能材料和能量转化器件工程技术研究中心, 广东省软物质重点实验 室, 广州 510006; 2. 广东环境保护工程职业学院, 佛山 528216)

摘 要: 本文采用固相法在 $900 、 1000 、 1100$ 和 $1200^{\circ} \mathrm{C}$ 煅烧温度条件下合成了 $\mathrm{Li}_{0.33} \mathrm{La}_{0.56} \mathrm{TiO}_{3}(\mathrm{LLTO})$ 固态电解质材 料, 并将其组装为 LLTO 固态离子电容器。采用 X射线衍射(XRD)、扫描电子显微镜(SEM)、X射线光电子能谱(XPS)、 电化学阻抗谱(EIS)和循环伏安法(CV)等技术研究了煅烧温度对 LLTO 固态电解质和固态离子电容器的显微结构、 形貌、离子电导率和储能性能的影响。实验表明, 较高的煅烧温度有利于获得性能优异的 LLTO 固态离子电容器。 在室温下， $1200^{\circ} \mathrm{C}$ 煅烧温度制备的固态离子电容器晶粒离子电导率高达 $9.6 \times 10^{-4} \mathrm{~S} / \mathrm{cm}$, 且具有明显的双电层电容 特性, 在 $4 \mathrm{~V}$ 电压窗口下比电容为 $3.52 \mathrm{mF} / \mathrm{g}$ 。此外, 固态离子电容器比电容随晶粒电导率的增大而增大, 同时受电 极与固态电解质接触面积的影响。

关 键 词: 钛酸镧锂(LLTO); 固态电解质; 离子电导率; 固态离子电容器

中图分类号: TQ174; TM53 文献标识码: A

\section{Influence of Calcining Temperature on the Property of $\mathrm{Li}_{0.33} \mathrm{La}_{0.56} \mathrm{TiO}_{3}$ Solid-state Ionic Capacitor}

\author{
LU Dong-Liang $^{1,2}$, DAI Guang-Zhou ${ }^{1}$, YAO Ying-Bang ${ }^{1}$, TAO Tao ${ }^{1}$, LIANG Bo ${ }^{1}$, LU Sheng-Guo ${ }^{1}$
}

(1. Guangdong Provincial Key Laboratory of Functional Soft Condensed Matter, Guangdong Provincial Research Center on Smart Materials and Energy Conversion Devices, School of Materials and Energy, Guangdong University of Technology, Guangzhou 510006, China; 2. Guangdong Polytechnic of Environmental Protection Engineering, Foshan 528216, China)

\begin{abstract}
Li}_{0.33} \mathrm{La}_{0.56} \mathrm{TiO}_{3}$ was synthesized under different calcination temperatures by using solid-state reaction method and assembled as LLTO solid-state ionic capacitors in this study. X-ray powder diffraction (XRD), scanning electron microscope (SEM), X-ray photoelectron spectroscopy (XPS), electrochemical impedance spectroscopy (EIS), and cyclic voltammetry (CV) were employed to investigate the influence of calcination temperature on the microstructure, morphology, ionic conductivity, and the energy storage property of the as-prepared products. Results indicate that $\mathrm{Li}_{0.33} \mathrm{La}_{0.56} \mathrm{TiO}_{3}$ calcined at higher temperature exhibited better performance, and the product synthesized at $1200^{\circ} \mathrm{C}$ occupied the highest grain ionic conductivity $\left(9.6 \times 10^{-4} \mathrm{~S} / \mathrm{cm}\right)$ with obvious double-layer capacitor characteristics, while the specific capacitor is nearly $3.52 \mathrm{mF} / \mathrm{g}$ at a $4 \mathrm{~V}$ potential window. Besides, the specific capacitor of the solid-state capacitor increases with the increment of grain ionic conductivity and effected by the contact area between electrode and solid electrolyte.
\end{abstract}

Key words: lithium lanthanum titanate (LLTO); solid-state electrolyte; ionic conductivity; solid-state ionic capacitor

收稿日期：2018-01-29; 收到修改稿日期：2018-04-03

基金项目: 国家自然科学基金(51372042); 广东省自然科学基金重大基础研究培育项目(2015A030308004); 国家自然科学 基金委-广东省联合基金(U1501246)

Natural Science Foundation of China (51372042); The Guangdong Provincial Natural Science Foundation (2015A030308004); The NSFC-Guangdong Joint Fund (U1501246)

作者简介：卢东亮(1985-), 男, 博士研究生. E-mail: 327560923@qq.com

通讯作者：鲁圣国，教授. E-mail: sglu@gdut.edu.cn 
由于石油资源日趋短缺, 同时, 传统然油内燃 机尾气排放对环境的污染越来越严重, 对人体造成 很大的影响, 新型环保能源装置成为研究热点 ${ }^{[1-2]}$ 。 世界各国已经进行混合动力、燃料电池、化学电池 产品及应用的研究与开发, 取得了一定的成效。但 是由于它们固有的使用寿命短、温度特性差、化学 电池污染环境、系统复杂、造价高昂等致命弱点, 一 直没有很好的解决办法。而超级电容器以其优异的 特性扬长避短, 可以部分或全部替代传统的化学电 池用作车辆的牵引电源和启动能源, 并且具有比传 统的化学电池更加广泛的用途 ${ }^{[3]}$ 。

常规超级电容器的材料主要包括电极材料、电 解质材料、集流体材料和隔膜材料等。根据电解质 材料的不同, 可将电容器分为液态超级电容器和固 态超级电容器。液态超级电容器一般包括水系电解 液和有机电解液两种, 二者的电导率均较高, 但分 解电压较低, 且有挥发性、污染性和不稳定性, 这些 限制了该类型超级电容器的推广应用。相比之下, 固态离子电容器采用固态电解质、电介质或复合材 料, 可提升电容器的工作电压。同时, 由于省去了隔 膜, 不仅降低了封装工艺的难度, 也有利于制备柔 性或多层固体离子电容器。此外, 采用固态电解质 还可以解决液态电解质泄露、挥发等安全性问题, 特别适用于新能源电动车。

在所研究的固态电解质中, 钙钛矿结构的 $\mathrm{Li}_{3 x} \mathrm{La}_{(2 / 3-x)} \mathrm{TiO}_{3}$ (LLTO) 备受关注, 因为它在室温下 具有较高的离子晶粒电导率, 约为 $10^{-3} \mathrm{~S} / \mathrm{cm}^{[4-6]}$ 。研 究者针对 LLTO 在固态锂离子电池的应用进行了大 量研究, 而将其制备为离子电容器的研究较少。本 实验中, 采用固相法合成 LLTO 固态电解质, 并制 备了 LLTO 固态离子电容器。探究不同㷽烧温度对 LLTO 固态离子电容器电性能和储能性能影响。

\section{1 实验方法}

\subsection{LLTO 固态电解质的制备}

实验所用原料为分析纯 $\mathrm{TiO}_{2} 、 \mathrm{Li}_{2} \mathrm{CO}_{3}$ 和 $\mathrm{La}_{2} \mathrm{O}_{3}$ 。 称量前先将 $\mathrm{La}_{2} \mathrm{O}_{3}, \mathrm{TiO}_{2}$ 分别在 $1200,800^{\circ} \mathrm{C}$ 保温 $4 \mathrm{~h}$, 以除去水和其它吸附物。固态电解质采用固相反应 合成。根据 $\mathrm{Li}_{3 x} \mathrm{La}_{2 / 3-x} \mathrm{TiO}_{3}$ 化学计量比 $(x=0.11)$ 称取 一定量的原料 $\mathrm{La}_{2} \mathrm{O}_{3}, \mathrm{Li}_{2} \mathrm{CO}_{3}, \mathrm{TiO}_{2}$ 。将所称取的原料 加入行星式球磨机, 在无水乙醇中球磨 $15 \mathrm{~h}$ 。之后 将所得样品置于烘箱中在 $80^{\circ} \mathrm{C}$ 干燥 $5 \mathrm{~h}$, 干燥研磨 后的样品在 $900 、 1000 、 1100$ 和 $1200^{\circ} \mathrm{C}$ 四个温度条 件下煅烧 $12 \mathrm{~h}$ 得到煅烧粉体。

\section{2 固态离子电容器制备}

将制备的 LLTO 㷽烧粉体在室温、5 MPa 的压 力下, 采用压片成型制备成坏体, 之后在 $1350^{\circ} \mathrm{C}$ 烧 结并保温 $6 \mathrm{~h}$, 制成直径约 $10 \mathrm{~mm}$, 厚度约 $0.6 \mathrm{~mm}$ 的陶瓷样品。在烧结过程中, 样品表面覆盖煆烧粉 体防止 $\mathrm{Li}$ 挥发。采用真空离子溅射镀膜设备在陶瓷 样品表面浌射 $\mathrm{Au}$ 电极, 制备成固态离子电容器。

\section{3 实验表征方法}

采用 X 射线衍射仪 $(X R D$, 德国 Bruker D8 Advance)对材料的晶体结构进行表征; 采用扫描电镜 (SEM, 德国 Carl Zeiss ZEISS Ultra 55)对样品的形 貌进行观察。采用 $X$ 射线光电子能谱(XPS, 美国 Thermo Fisher ESCALAB 250Xi)对元素价态进行测 定, 采用交流阻抗法(EIS, 瑞士万通 Autolab 电化学 工作站)测试所得样品的电导率, 并采用循环伏安 法 $(\mathrm{CV}$, 上海辰华 $\mathrm{CHI} 600 \mathrm{E})$ 测试样品的电容。

\section{2 结果与讨论}

\section{1 炦烧温度对材料晶体结构的影响}

图 1 为不同煅烧温度条件下合成的 LLTO XRD 图谱。通过对 XRD 图谱分析, 全部样品均为立方相, $900^{\circ} \mathrm{C}$ 炦烧粉体的晶格参数 $a=b=c=0.38694 \mathrm{~nm}$, 晶 胞体积为 $0.0579323 \mathrm{~nm}^{3} ; 1000^{\circ} \mathrm{C}$ 㷽烧粉体的晶格参 数 $a=b=c=0.38711 \mathrm{~nm}$, 晶胞体积为 $0.0580120 \mathrm{~nm}^{3}$; $1100^{\circ} \mathrm{C}$ 煅烧粉体晶格参数 $a=b=c=0.38664 \mathrm{~nm}$, 晶胞 体积为 $0.0577980 \mathrm{~nm}^{3} ; 1200^{\circ} \mathrm{C}$ 煅烧粉体晶格参数 $a=b=c=0.38672 \mathrm{~nm}$, 晶胞体积为 $0.0578342 \mathrm{~nm}^{3[7-8]}$ 。

通过对不同煅烧温度所合成粉体的 XRD 图谱 分析，在 $900^{\circ} \mathrm{C}$ 炦烧条件下，合成的样品中有较多 的 $\mathrm{Ti}$ 和 $\mathrm{La}$ 的氧化物, 存在较多杂相; 在 $1000^{\circ} \mathrm{C}$ 㷽烧条件下，合成的粉体中杂相明显减少; 随着 炦烧温度进一步升高，杂相相应减少，在 1100 、

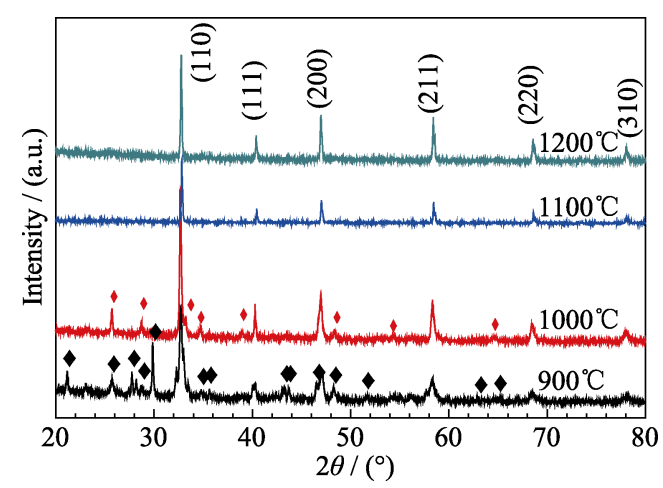

图 1 不同煅烧温度合成的 LLTO 固态电解质的 XRD 图谱 Fig. 1 XRD patterns of LLTO synthesized at different calcination temperatures 
$1200^{\circ} \mathrm{C}$ 煅烧条件下得到高纯度的 LLTO。相对而言, 在 $1100^{\circ} \mathrm{C}$ 炦烧得到的 LLTO 晶胞体积相对较小, $1200^{\circ} \mathrm{C}$ 煅烧条件下合成的 LLTO 晶胞体积相对较大, 更有利于 $\mathrm{Li}^{+}$的传输 ${ }^{[9-11]}$ 。

\section{2 煅烧温度对 LLTO 陶瓷形貌的影响}

㷽烧温度对 LLTO 固态电解质表面形貌的影响 如图 2 所示。由图 2(a)可以看出, 在 $900^{\circ} \mathrm{C}$ 炦烧条件 下制备的 LLTO 以立方相的晶粒为主, 晶粒较为致 密, 大小不均, 且平均粒径较小, 部分晶粒为不规 则形状。说明在低温㷽烧条件下得到的 LLTO 中存 在一定量的杂相。图 2(b)中 LLTO 的晶粒均匀性有 所提高, 以立方晶粒为主, 但存在个别四方相晶粒 且体积较大, 晶粒平均尺寸明显增大。说明 $1000^{\circ} \mathrm{C}$ 烣烧温度条件下杂相在一定程度上有所降低, 但根 据之前的 XRD 结果, 材料中又出现了新的杂相。由 图 2(c)和(d)可以看出, 在 1100 和 $1200^{\circ} \mathrm{C}$ 炦烧条件下, LLTO 晶粒较为均匀, 晶粒尺寸较为一致, 杂相较 少。相比较而言, 在 $1200^{\circ} \mathrm{C}$ 煅烧得到的 LLTO 晶粒 尺寸比 $1100^{\circ} \mathrm{C}$ 条件有所增大。同时, 由图 2 可见, 在 $1100^{\circ} \mathrm{C}$ 煅烧制备而成的 LLTO 陶瓷样品致密度相对 较低。

\section{3 煅烧温度对载流子浓度的影响}

图 3 显示了 900 和 $1200^{\circ} \mathrm{C}$ 炦烧条件下制备的 LLTO 粉体主要元素的 XPS 图谱。图 3(a)是 900/ $1200^{\circ} \mathrm{C}$ 传烧条件下制备的 LLTO 粉体的 Ti2p 核图谱。 所有的样品都在 $458 \mathrm{eV}$ 键能附近具有一个 $\mathrm{Ti}^{4+} 2 \mathrm{p}_{3 / 2}$ 的峰, 同时在 $464 \mathrm{eV}$ 键能附近具有一个 $\mathrm{Ti}^{4+} 2 \mathrm{p}_{1 / 2}$ 的 肩峰。在 $\mathrm{Ti}^{4+} 2 \mathrm{p}_{3 / 2}$ 和 $\mathrm{Ti}^{4+} 2 \mathrm{p}_{1 / 2}$ 峰的低键能侧均无其 他肩峰出现, 说明合成的样品中不存在 $\mathrm{Ti}^{3+}$ 离子 ${ }^{[12]}$ 。

图 3(b)是 $900 / 1200^{\circ} \mathrm{C}$ 炦烧条件下制备的 LLTO 粉体的 La3d 核图谱。由于 La3d 自旋轨道的分裂,
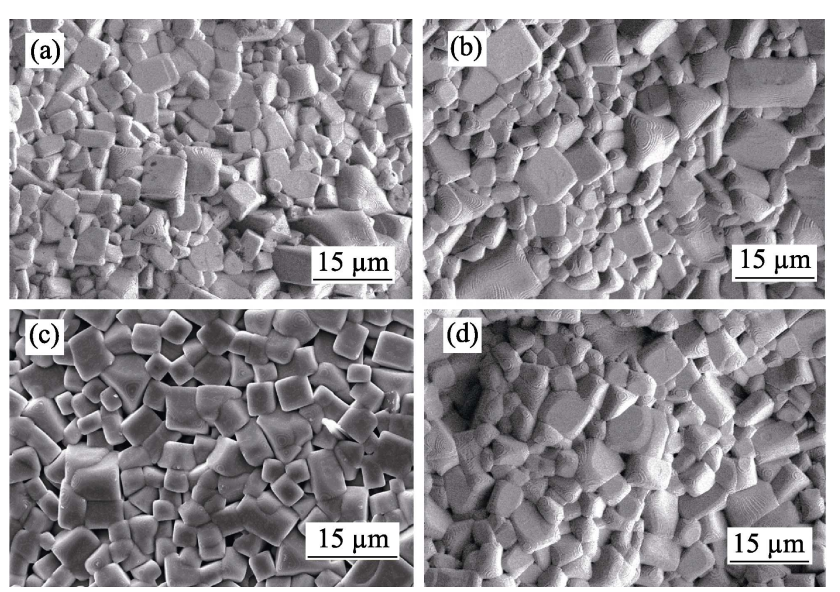

图 2 由不同煅烧温度制备的 LLTO 陶瓷样品的 SEM 照片 Fig. 2 SEM images of LLTO ceramics specimens prepared at different calcining temperatures

(a) $900^{\circ} \mathrm{C}$; (b) $1000^{\circ} \mathrm{C}$; (c) $1100^{\circ} \mathrm{C}$; (d) $1200^{\circ} \mathrm{C}$
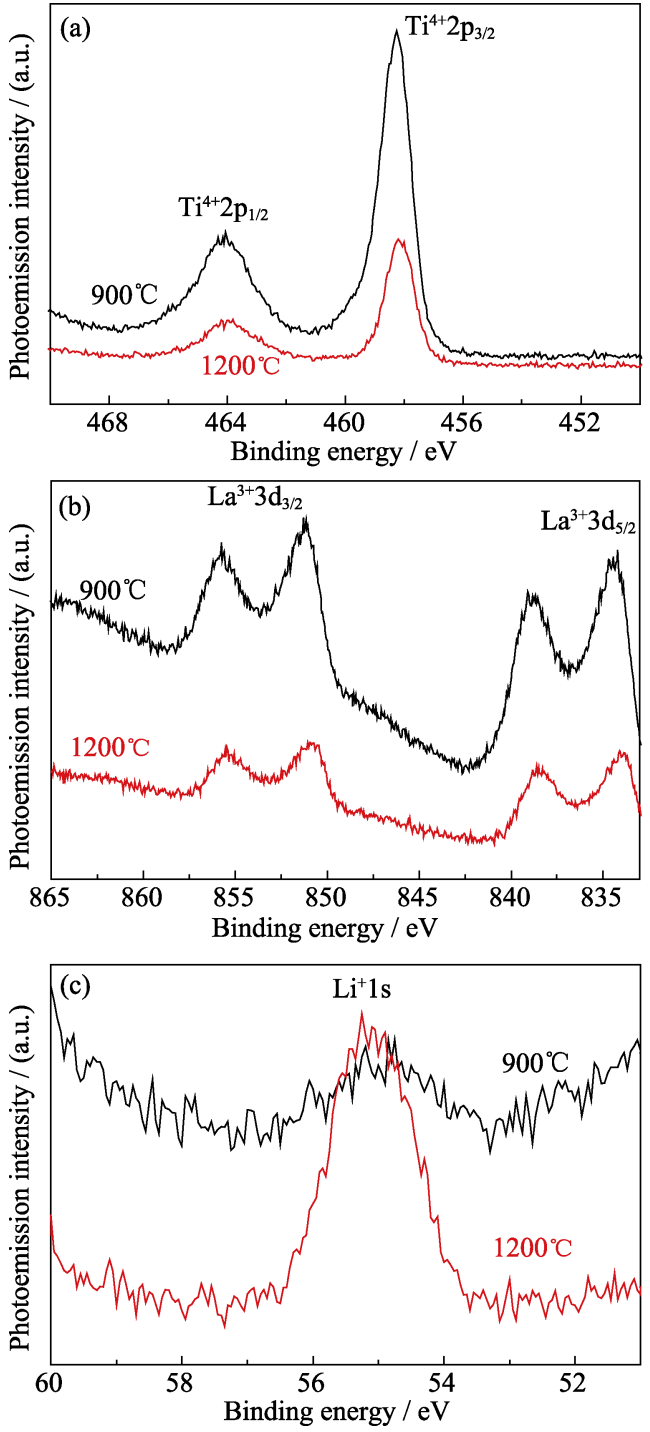

图 3900 和 $1200^{\circ} \mathrm{C}$ 煅烧合成的 LLTO 粉体主要元素的 XPS 图谱

Fig. 3 XPS spectra of main elements in the LLTO powders calcined at $900^{\circ} \mathrm{C}$ and $1200^{\circ} \mathrm{C}$

(a) Titanium; (b) Lanthanum; (c) Lithium

$\mathrm{La}^{3+} 3 \mathrm{~d}_{5 / 2}$ 和 $\mathrm{La}^{3+} 3 \mathrm{~d}_{3 / 2}$ 分别对应产生两个峰值。此外, 在 $\mathrm{La}^{3+} 3 \mathrm{~d}_{5 / 2}$ 和 $\mathrm{La}^{3+} 3 \mathrm{~d}_{3 / 2}$ 峰值外存在两个伴峰 ${ }^{[12-14]}$ 。

图 3(c)是 $900 / 1200^{\circ} \mathrm{C}$ 㷽烧条件下制备的 LLTO 粉体的 Li1s 核谱图。不同煅烧温度下制备的样品的 峰强有明显的不同。900 ${ }^{\circ} \mathrm{C}$ 条件下制备的 LLTO 粉体 的 Li1s 峰值强度明显低于 $1200^{\circ} \mathrm{C}$ 条件下制备的样 品。这说明 $1200^{\circ} \mathrm{C}$ 条件下制备的样品中 $\mathrm{Li}^{+}$的数量 较 $900^{\circ} \mathrm{C}$ 条件下制备的样品明显增多。在 $900^{\circ} \mathrm{C}$ 条件 下，虽然煅烧温度较低，但没能完全合成 LLTO 材 料，因而炦烧过程中不可避免地产生了 $\mathrm{Li}^{+}$损失。而 在 $1200^{\circ} \mathrm{C}$ 条件下反应生成的 LLTO 材料, 降低了因 挥发产生的 $\mathrm{Li}^{+}$损失, 所以 $\mathrm{Li}^{+}$离子的数量较多。在 XPS 图谱中, Li1s 峰值未出现在 $54 \mathrm{eV}$ 处, 而是处于 $55 \mathrm{eV}$ 附近, 亦能说明该现象 ${ }^{[12]}$ 。 


\section{4 煅烧温度对固态离子电容器离子导电性 能的影响}

图 4 为不同耘烧温度制备的 LLTO 陶瓷样品的 交流阻抗谱图。交流阻抗曲线由在高频区的半圆和 低频区的斜线组成。半圆对应于陶瓷样品中的离子 电导, 而斜线则是由于在金电极表面产生极化所形 成的电导所致。图 4 插图为对应的等效电路, 该等 效电路将晶粒和晶界相对应的两个阻抗部分进行串 联; 晶粒部分以电阻为主, 忽略电容, 用电阻 $R_{\mathrm{b}}$ 表 示; 而晶界部分既有电阻也有电容, 其阻抗由电阻 $R_{\mathrm{gb}}$ 和相位角 $\mathrm{CPE}_{\mathrm{gb}}$ 表示; 电极的阻抗采用另外一个 相位角 $\mathrm{CPE}_{\mathrm{e}}$ 表示 ${ }^{[12]}$ 。通过拟合计算得到 LLTO 样 品的晶粒和晶界电阻, 并根据样品的厚度和电极面 积计算得到材料的电导率。

对不同煅烧温度制备的 LLTO 固态离子电容器 的离子体电导率和晶界电导率分别进行计算, 并将 结果总结在图 5 中。其中 $1200^{\circ} \mathrm{C}$ 㷽烧制备的固态离 子电容器具有最高的晶粒电导率和晶界电导率, 分 别为 $9.6 \times 10^{-4} \mathrm{~S} / \mathrm{cm}$ 和 $2.32 \times 10^{-5} \mathrm{~S} / \mathrm{cm}$, 较其它煅烧

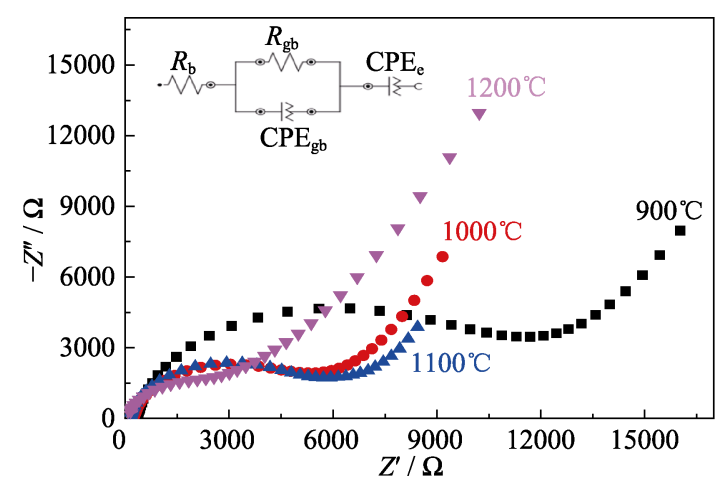

图 4 不同㷽烧温度制备的 LLTO 固态离子电容器的交流阻 抗谱图, 插图为等效电路图

Fig. 4 Typical EIS of the LLTO solid-state ion capacitors with different calcination temperatures with inset showing equivalent circuit

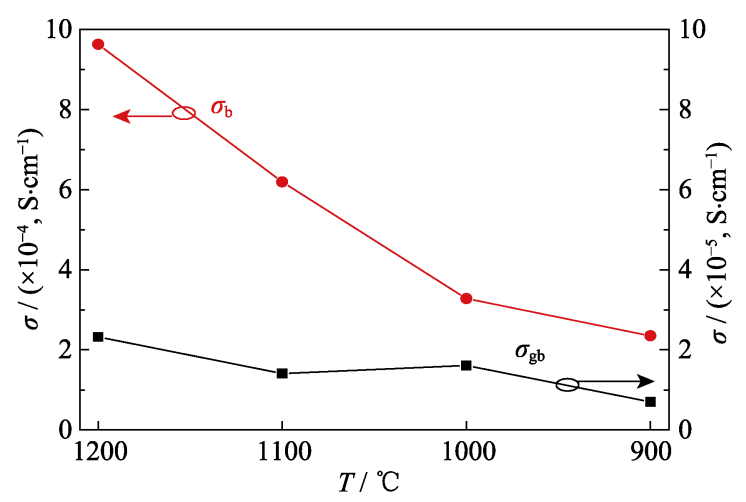

图 5 室温下晶粒电导率和晶界电导率随炦烧温度的变化

Fig. 5 Grain and grain boundary conductivities as a function of calcination temperature at room temperature
温度制备的样品明显增大。总体而言，随着敾烧温 度的升高, 制备的 LLTO 固态离子电容器总电导率 基本呈现增大趋势。从图中可以看到，相对于晶粒 体电导率, 晶界电导率明显低很多。晶界的存在阻 隔了 $\mathrm{Li}^{+}$在晶粒晶格间传输的通道, 所以晶界本身 是影响 $\mathrm{Li}^{+}$迁移的壁垒。其它因素如晶界处的气孔、 杂相等, 也会使得晶界电导率进一步降低。

晶粒的离子体电导率随着煅烧温度的降低而降 低。这与前面 XRD 分析的结果相吻合, 说明在较高 的衫烧温度下合成的 LLTO 结晶性更好, 纯度更高。 同时， $1100^{\circ} \mathrm{C}$ 煅烧条件下制备的陶瓷样品晶界电导 率比 $1000^{\circ} \mathrm{C}$ 炦烧条件明显减小, 这与上面 SEM 的 分析相符合。虽然在 $1100^{\circ} \mathrm{C}$ 条件下得到的样品的晶 粒生长较好, 但制备的陶瓷样品致密度不高, 而且 晶界处存在少量的气孔，导致该温度条件下制备的 样品的晶界电导率较小。

\section{5 煅烧温度对 LLTO 固体离子电容器储能 性能的影响}

不同煅烧温度合成的 LLTO 粉体经过压片、烧 结和电极制备制作成固态离子电容器, 如图6 所示。 固态离子电容器的储能主要通过在外加电场作用下, 固态电解质内 $\mathrm{Li}^{+}$和空位的定向迁移, 形成双电层。 采用电化学工作站在不同电压窗口下对其循环伏安 特性进行测试, 扫速为 $1 \mathrm{mV} / \mathrm{s}$ 。结果如图 7 所示。 从图 7(a) (d)的循环伏安特性测试结果来看, 除了 900 和 $1000^{\circ} \mathrm{C}$ 煅烧温度条件外, 其它温度条件下合 成的样品在-1.5 1.5 V 之间的循环伏安测试结果比 较稳定, 形状趋向矩形, 说明在该电压窗口区间内 其双电层电容行为明显, 没有氧化还原峰说明界面 处没有发生反应。

当电位测试区间在 $-2 \sim 2 \mathrm{~V}$ 时, 固态离子电容器 循环伏安曲线上出现了氧化还原峰。这说明在 $3 \mathrm{~V}$ 电压窗口下该电容器具有较好的双电层电容行为, 当电压窗口增大时, $\mathrm{Li}^{+}$在固态电解质和 $\mathrm{Au}$ 电极界面

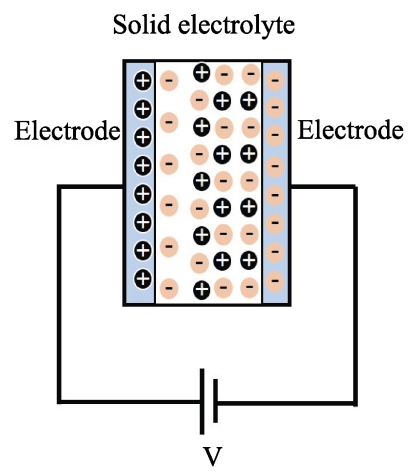

图 6 固态离子电容器示意图

Fig. 6 Schematic diagram for a solid-state ionic capacitor 

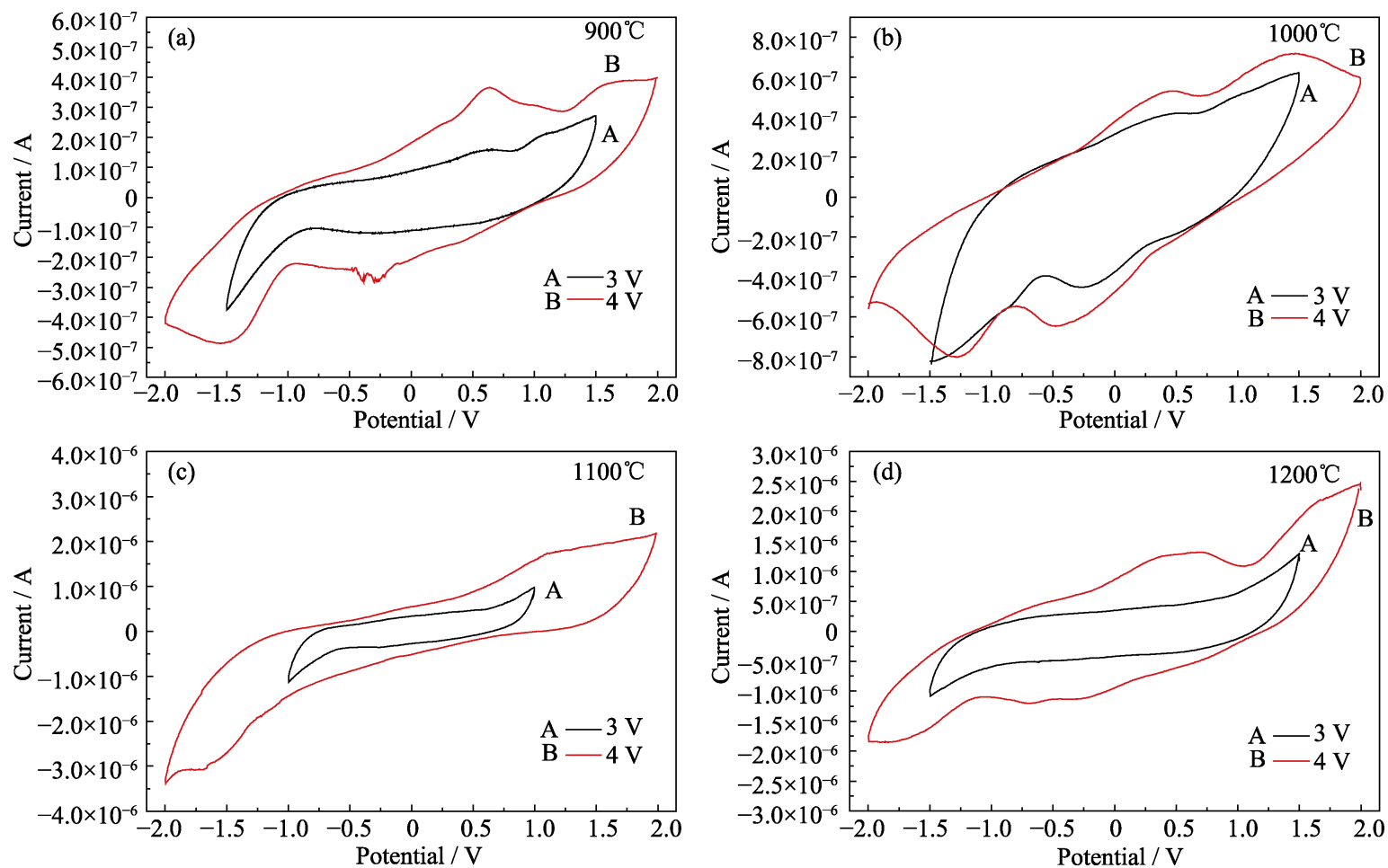

图 7 不同煅烧温度制备的固态离子电容器在不同电压窗口下的循环伏安曲线

Fig. 7 The cyclic voltammograms for solid-state ion capacitors recorded under different potential windows (a) $900^{\circ} \mathrm{C}$; (b) $1000^{\circ} \mathrm{C}$; (c) $1100^{\circ} \mathrm{C}$; (d) $1200^{\circ} \mathrm{C}$

处发生了氧化还原反应，产生了一定的赝电容行为。

将不同煅烧温度条件制备的电容器在 $-2 \sim 2 \mathrm{~V}$ 区间内的比电容进行计算，结果列于表 1 中。可见， 由 $1200^{\circ} \mathrm{C}$ 㷽烧制备的固态离子电容器具有相对较 高的比电容, 约为 $3.52 \mathrm{mF} / \mathrm{g}$, 而其它㷽烧温度下所 制备样品的比电容则更小一些。比电容大小与晶粒 离子电导率有关, 即随着晶粒电导的增大, 固态离 子电容器的比电容随之提升。

同时, 从结果分析, 1100 和 $1200^{\circ} \mathrm{C}$ 炦烧条件下 的晶粒电导率相差值较大, 而比电容并未有太大变 化。由上面 SEM 照片分析可知, 原因可能在于在 $1100^{\circ} \mathrm{C}$ 煅烧制备的 LLTO 陶瓷表面不够致密，存在一 些空隙, 相应增加了 LLTO 陶瓷表面的比表面积。这

\section{表 1 不同煅烧温度制备的 LLTO 固态离子电容器 在-2 2 V 区间内的比电容}

Table 1 Specific capacitance of the LLTO solid-state ionic capacitors with different calcination temperatures under a potential range of $-2 \mathrm{~V}-2 \mathrm{~V}$

\begin{tabular}{ccc}
\hline Samples & Calcination temperature $/{ }^{\circ} \mathrm{C}$ & Specific capacitance $/\left(\mathrm{mF} \cdot \mathrm{g}^{-1}\right)$ \\
\hline 1 & 900 & 0.79 \\
2 & 1000 & 1.51 \\
3 & 1100 & 3.29 \\
3 & 1200 & 3.52 \\
\hline
\end{tabular}

些空隙扩大了 $\mathrm{Au}$ 电极与 LLTO 固态电解质的界面接 触面积, 进而提高了电容器的比电容。因此, 可以初 步推断固态离子电容器较大的比电容主要来自于两 个方面的共同作用: 1)固态电解质的晶粒离子电导率 较高; 2)固态电解质与电极界面接触面积较大。

\section{3 结论}

1)通过高温固相法合成了 $\mathrm{Li}_{0.33} \mathrm{La}_{0.56} \mathrm{TiO}_{3}$ 钙钛 矿型固态电解质，合成的 LLTO 为立方晶型。炦烧 温度对材料的制备有较大的影响，在 $1200^{\circ} \mathrm{C}$ 衫烧温 度条件下, 合成了高纯度、少杂相的 LLTO 固态电 解质。

2)由 $1200^{\circ} \mathrm{C}$ 煅烧条件制备的 LLTO 固态离子电 容器, 晶粒的形貌以立方和四方相为主, 表面致密, $\mathrm{Li}^{+}$浓度较高。在室温下, 该样品的晶粒电导率为 $9.6 \times 10^{-4} \mathrm{~S} / \mathrm{cm}$, 晶界电导率为 $2.32 \times 10^{-5} \mathrm{~S} / \mathrm{cm}$, 相对 其它煅烧条件的样品，离子电导率最高。

3)通过对 LLTO 固态离子电容器的电容性能测 试, 表明该类电容器在 $3 \mathrm{~V}$ 电压窗口下具有较好的 双电层储能特性, 而在 $4 \mathrm{~V}$ 以上时, 会出现一定的 赝电容行为。同时, 比电容大小与晶粒离子电导率 有关，随着电导率的增大而增大。在-2 2 V 测试区 
间，由 $1200^{\circ} \mathrm{C}$ 㷽烧条件制备的 LLTO 固态离子电容 器具有最大比电容 $3.52 \mathrm{mF} / \mathrm{g}$ 。

4)固态离子电容器的电容特性不仅与离子电导 率有关, 同时受电极与固态电解质接触面积的影响。

\section{参考文献:}

[1] 雷永泉, 万 群, 石永康. 新能源材料. 天津: 天津大学出版社, 2000: 1-10.

[2] BURKE A. Ultracapacitors: why, how, and where is the technology. Journal of Power Sources, 2000, 91(1): 37-50.

[3] CERICOLA D, KÖTZ R. Hybridization of rechargeable batteries and electrochemical capacitors: principles and limits. Electrochimica Acta, 2012, 72(4): 1-17.

[4] ZHANG BINGKAI, TAN RUI, YANG LUYI, et al. Mechanisms and properties of ion-transport in inorganic solid electrolytes. Energy Storage Materials, 2018, 10: 139-159.

[5] STRAMARE S, THANGADURAI V, WEPPNER W. Lithium lanthanum titanates: a review. Cheminform, 2003, 34(52): 3974-3990.

[6] LIAO C L, WEN C H, FUNG K Z. Preparation of RF-sputtered lithium cobalt oxide nanorods by using porous anodic alumina (PAA) template. Journal of Alloys and Compounds, 2006, 414(1/2): 302-309.

[7] IBARRA J, VÁREZ A, LEÓN C, et al. Influence of composition on the structure and conductivity of the fast ionic conductors
$\mathrm{La}_{2 / 3-} \mathrm{Li}_{3 x} \mathrm{TiO}_{3} \quad(0.03 \leqslant x \leqslant 0.167)$. Solid State Ionics, 2000, 134(3/4): 219-228.

[8] LE DINH TRONG, TRAN THI THAO, NGUYEN NANG DINHB. Characterization of the Li-ionic conductivity of $\mathrm{La}_{(2 / 3-x)} \mathrm{Li}_{3 x} \mathrm{TiO}_{3}$ ceramics used for all-solid-state batteries. Solid State Ionics, 2015, 278: 228-232.

[9] INAGUMA Y, CHEN L, ITOH M, et al. Candidate compounds with perovskite structure for high lithium ionic conductivity. Solid State Ionics, 1994, s70-71(94): 196-202.

[10] 郭晓娜. 基于 $\mathrm{Li}_{3 x} \mathrm{La}_{2 / 3-x} \mathrm{TiO}_{3}$ 的无机固态锂离子导体的电性能研 究. 武汉: 华中科技大学硕士学位论文, 2015.

[11] KIM I S, JUNG W H, INAGUMA Y, et al. Dielectric properties of a-site deficient perovskite-type lanthanum-calcium-titanium oxide solid solution system $\left[(1-x) \mathrm{La}_{2 / 3} \mathrm{TiO}_{3-x} \mathrm{CaTiO}_{3}(0.1 \leqslant x \leqslant 0.96)\right]$. Materials Research Bulletin, 1995, 30(3): 307-316.

[12] MEI AO, JIANGB QING HUI, LIN YUAN HUA, et al. Lithium lanthanum titanium oxide solid-state electrolyte by spark plasma sintering. Journal of Alloys and Compounds, 2009, 486(1): 871-875.

[13] YANG K Y, FUNG K Z, WANG M C. X-ray photoelectron spectroscopic and secondary ion mass spectroscopic examinations of metallic-lithium-activated donor doping process on $\mathrm{La}_{0.56} \mathrm{Li}_{0.33} \mathrm{TiO}_{3}$ surface at room temperature. Journal of Applied Physics, 2006, 100(5): 056102-1-3.

[14] PLYUTO I V, SHPAK A P, JERZY S, et al. XPS characterisation of carbon-coated alumina support. Surface and Interface Analysis, 2010, 38(5): 917-921. 